\title{
Trypanosoma brucei brucei: differences in the nuclear chromatin of bloodstream forms and procyclic culture forms
}

\author{
W. SCHLIMME, M. BURRI, K. BENDER, B. BETSCHART and H. HECKER* \\ Swiss Tropical Institute, Postfach CH-4402, Basel, Switzerland
}

(Received 9 December 1992 ; revised 1 March 1993; accepted 1 March 1993)

\section{SUMMARY}

Nucleosome filaments of two stages of the life-cycle of Trypanosoma brucei brucei, namely bloodstream forms and procyclic culture forms, were investigated by electron microscopy. Chromatin of bloodstream forms showed a salt-dependent condensation. The level of condensation was higher than that shown by chromatin from procyclic culture forms, but $30 \mathrm{~nm}$ fibres as formed in rat liver chromatin preparations were not found. Analysis of histones provided new evidence for the existence of $\mathrm{H} 1$-like proteins, which comigrated in the region of the core histones in SDS-PAGE and in front of the core histones in Triton acid urea gels. Differences were found between the H1-like proteins of the two trypanosome stages as well as between the core histones in their amount, number of bands and banding pattern. It can be concluded that T. b. bruce $i$ contains a full set of histones, including $\mathrm{H} 1$-like proteins, and that the poor condensation of its chromatin is not due to the absence of $\mathrm{H} 1$, but most probably due to histone-DNA interaction being weak. It is obvious that structural and functional differences of the chromatin exist not only between T. b. brucei and higher eukaryotes, but also between various stages of the life-cycle of the parasite. It is therefore not adequate to investigate the chromatin only of the procyclic culture forms as a model for all stages of the life-cycle of $T . b$. brucei.

Key words: Trypanosoma brucei brucei, chromatin, histones, lower eukaryotes, bloodstream forms, procyclic culture forms.

\section{INTRODUCTION}

Trypanosomes, the protozoan parasites of man and animals, have various nuclear features different from those of higher eukaryotes. No condensed chromosomes can be visualized during nuclear division in trypanosomes (Vickerman \& Preston, 1970), and the compaction of the chromatin in the nucleus is distinctly less pronounced as compared to the chromatin of rat liver nuclei (Hecker \& Gander, 1985).

The chromatin of $T . b$. brucei and of $T$. cruzi procyclic culture forms was shown to be organized in a nucleosome filament-like form. However, the nucleosomes were spaced irregularly and no condensation into a typical $30 \mathrm{~nm}$ fibre took place under experimental conditions. The nuclear chromatin was digested rapidly by micrococcal nuclease, and the interactions between DNA and proteins were relatively weak and were easily destabilized under experimental conditions, which are normally used for the isolation of chromatin from higher eukaryotes (Hecker \& Gander, 1985 ; Hecker et al. 1989; Bender, Betschart \& Hecker, $1992 c$ ). In addition, no histone H1 could be demonstrated (Hecker \& Gander, 1985 ; Hecker et al. 1989). Other workers also postulated that H1 was absent (Bender et al. 1992a,b,c,d). However, these results are controversial. Recent

* Reprint requests to Professor H. Hecker. studies demonstrated $\mathrm{H} 1$-like proteins in $T$. cruzi and Crithidia fasciculata (Toro \& Galanti, 1988, 1990; Duschak \& Cazzulo, 1990). These histones showed biochemical properties similar to those of histones from Tetrahymena fasciculata (Johmann \& Gorovsky, 1976).

On the basis of the migration pattern in various gel systems and amino acid sequence analysis, Bender $e t$ $a l$. $(1992 a, b)$ were able to show that the four core histones of T.b.brucei procyclic culture forms, namely a, b, c, d are the counterparts of $\mathrm{H} 3, \mathrm{H} 2 \mathrm{~B}$, $\mathrm{H} 2 \mathrm{~A}$ and $\mathrm{H} 4$ of higher eukaryotes. The core histones of higher eukaryotes are among the most conserved proteins known. Among the histones, $\mathrm{H} 4$ and $\mathrm{H} 3$ are the best conserved, followed by $\mathrm{H} 2 \mathrm{~A}, \mathrm{H} 2 \mathrm{~B}$ and $\mathrm{H} 1$ (van Holde, 1989). Biochemical differences between the core histones of T.b.brucei procyclic culture forms and the core histones of higher eukaryotes were found by Bender et al. $(1992 a, b)$. Differences of about $35 \%$ exist in the amino acid sequence of the $\mathrm{N}$ - and $\mathrm{C}$-terminal regions of the histone $\mathrm{H} 4$ of calf thymus and trypanosomes (Bender et al. 1992b; Toro et al. 1992).

All previous investigations have been carried out with $T . b$. brucei procyclic culture forms which can be cultivated to a density of $3 \times 10^{7} / \mathrm{ml}$ (Brun \& Schönenberger, 1979). The in vitro cultivation of bloodstream forms, the parasitic stage affecting mammals, is possible only to a density of $2 \times 10^{6} / \mathrm{ml}$ (Hamm et al. 1990). Therefore studies on the 
chromatin of this stage depend on its propagation in laboratory animals. It was not known, whether the differences found between the chromatin of procyclic culture forms and that of higher eukaryotes would also be found in bloodstream forms.

In the present study, the chromatin of procyclic culture forms and bloodstream forms of $T . b$. bruce $i$ was compared with rat liver chromatin, using a variety of new separation techniques and staining methods. Also electron microscopy (EM) and enzymic digestion were used to find out whether there were differences between the chromatin of the two forms, and between that of bloodstream forms and higher eukaryotes. The validity of taking the chromatin of procyclic culture forms as a model for all the stages of the life-cycle of $T . b$.brucei was evaluated.

\section{MATERIALS AND METHODS}

Livers were from rats from the SIV IVANOVAS strain. Trypanosoma b. bruce $i$ STIB 345 AB procyclic culture forms, cultivated in SDM 79 medium containing $10 \%$ heat-inactivated foetal bovine serum, $10 \mu \mathrm{g} / \mathrm{ml}$ haemin and $10 \mu \mathrm{g} / \mathrm{ml}$ gentamicin at $27^{\circ} \mathrm{C}$ were used (Brun \& Schönenberger, 1979). Bloodstream forms of STIB $345 \mathrm{AB}$ were grown in SIV rats. Rats were inoculated intraperitoneally with $10^{6}$ trypanosomes, and killed on day 5 .

\section{Investigation of the influence of the temperature on the chromatin of procyclic culture forms}

To investigate the effect of temperature, procyclic culture forms were cultivated as described above, but incubated for 1 week at $32^{\circ} \mathrm{C}$. After that period they were incubated at the following temperatures: $34,35,35.5,36,37^{\circ} \mathrm{C}$ for various periods of time.

\section{Purification of nuclei}

Approximately $10 \mathrm{~g}$ liver from rats was homogenized in the presence of $1 \mathrm{~mm}$ phenylmethylsulphonylfluoride (PMSF) and the nuclei were purified by centrifugation through sucrose cushions (Thoma, Koller \& Klug, 1979; Hecker \& Gander, 1985). T. $b$. bruce $i$ STIB $345 \mathrm{AB}$ were harvested, and $2-3 \times 10^{10}$ exponentially growing procyclic cells resuspended in hypotonic buffer containing $0.5 \mathrm{M}$ 2-methyl-2,4pentandiol (hexylene glycol), $2.5 \mathrm{mM} \mathrm{CaCl}_{2}, 1 \mathrm{~mm}$ PMSF. They were lysed by nitrogen cavitation at 25 bar (Shapiro \& Doxsey, 1982), vortexed for $30 \mathrm{~s}$ and the isolated nuclei were washed in $90 \mathrm{~mm}$ suspension buffer, pH $7 \cdot 4$, containing $0 \cdot 1 \mathrm{~mm}$ disodium ethylendiaminetetraacetic acid $\left(\mathrm{Na}_{2} \mathrm{EDTA}\right)$ (Thoma et al. 1979; Hecker \& Gander, 1985).

Rats were anaesthetized with methoxyfluorane. Bloodstream forms were collected by cardiac puncture and separated from the blood by a DEAE-52 cellulose column (Lanham \& Godfrey, 1970). Trypanosomes were isolated by centrifugation at $1800 \mathrm{~g}$ for $20 \mathrm{~min}$. Isolation of the nuclei was the same from this step on as for procyclic culture forms.

\section{Preparation of soluble chromatin}

Nuclei of T. b. brucei were digested with 0.2 units of micrococcal nuclease (Sigma, N-3755) per $20 \mathrm{~A}_{260}$ at $30{ }^{\circ} \mathrm{C}$ for $50 \mathrm{~s}$. Nuclei of rat liver were digested with 0.4 units of micrococcal nuclease per $20 \mathrm{~A}_{260}$ at $37^{\circ} \mathrm{C}$ for $50 \mathrm{~s}$. The nuclei were centrifuged, and the chromatin solubilized by nuclear lysis in a hypotonic buffer containing $1 \mathrm{~mm}$ triethanolamine hydrochloride (TEACl) and $0 \cdot 2 \mathrm{~mm} \mathrm{Na}_{2}$ EDTA, $\mathrm{pH} 7 \cdot 4$. Insoluble material was removed by centrifugation (Thoma et al. 1979; Hecker \& Gander, 1985). The preparation of soluble chromatin was identical for procyclic culture forms and for bloodstream forms.

\section{Gradient analysis of the chromatin digest}

Gradient analysis was done in 5.5-28.5\% (w/v) $17 \mathrm{ml}$ isokinetic sucrose gradients containing $5 \mathrm{~mm}$ TEACl, pH 7.4, 0.2 $\mathrm{mM} \mathrm{Na}_{2}$ EDTA and $10 \mathrm{~mm} \mathrm{NaCl}$ (Noll, 1969). Centrifugation was performed for $14 \mathrm{~h}$ at $25000 \mathrm{~g}$ in a Kontron TS'T 28/17 swing-out rotor. The gradients were monitored at $254 \mathrm{~nm}$ and the bottom fractions containing the larger fragments of soluble chromatin were used for analysis by electron microscopy (Thoma \& Koller, 1981).

\section{Electron microscopy}

The fractions with the large chromatin fragments were divided into 4 aliquots and dialysed against $5 \mathrm{~mm}$ TEACl (pH 7.4), $0.2 \mathrm{~mm} \mathrm{Na}_{2}$ EDTA containing $0,10,40$ or $100 \mathrm{~mm} \mathrm{NaCl}$ respectively for $4 \mathrm{~h}$. Glutaraldehyde was then added to the dialysis buffer at a concentration of $0.2 \%(\mathrm{v} / \mathrm{v})$ for trypanosome chromatin or $0.1 \%$ for rat liver chromatin, and samples were fixed for at least $15 \mathrm{~h}$ and prepared for EM observation (Thoma et al. 1979). The concentration of $0.2 \%$ glutaraldehyde for trypanosomes was chosen after an experimental investigation of the effect of different levels of glutaraldehyde.

\section{Protein extraction from purified nuclei}

Nuclei were resuspended in $0.25 \mathrm{M} \mathrm{HCl}$ (Elpidina, Zaitseva \& Krasheninnikov, 1979) or in $5 \%(\mathrm{v} / \mathrm{v})$ perchloric acid (PCA) or $350 \mathrm{~mm} \mathrm{NaCl}$ in $10 \mathrm{~mm}$ TEACl, pH 7.4, (Sanders, 1977) and proteins extracted for $60 \mathrm{~min}$ under constant agitation. Insoluble material was pelleted at $4000 \mathrm{~g}$ for $5 \mathrm{~min}$. The supernatant fractions, containing the histones or non-histones, were removed and dialysed against $1 \mathrm{~mm}$ TEACl, $0 \cdot 2 \mathrm{~mm} \mathrm{Na}_{2}$ EDTA, $\mathrm{pH} \mathrm{7 \cdot 4}$, and lyophilized. 
All the preparations were carried out at $0-4{ }^{\circ} \mathrm{C}$ if not otherwise stated.

\section{Triton acid urea polyacrylamide gel electrophoresis}

Lyophilized proteins were dissolved in sample buffer containing $2.5 \mathrm{M}$ urea, $3 \%$ (v/v) 2-mercaptoethanol, $0.01 \%(\mathrm{v} / \mathrm{v})$ pyronine $\mathrm{G}, 0.9 \mathrm{M}$ acetic acid and incubated at $100{ }^{\circ} \mathrm{C}$ for $5 \mathrm{~min}$. The stacking gel contained $12 \%$, the resolution gel $15 \%$ polyacrylamide and both $2.5 \mathrm{M}$ urea, $0.9 \mathrm{M}$ acetic acid and $0.38 \%(\mathrm{v} / \mathrm{v})$ Triton DF 16 . After a first pre-run (25 $\mathrm{mA}$, up to constant voltage), a second pre-run was performed with $80 \mu \mathrm{l}$ of $1 \mathrm{M}$ cysteamine in $0.9 \mathrm{M}$ acetic acid per lane at $25 \mathrm{~mA}$ for $1 \mathrm{~h}$, to scavenge free radicals and to prevent oxidation of methionine residues. Then the probes were loaded and separated (Alfageme et al. 1974).

\section{SDS-tricine-PAGE}

Samples of proteins from soluble chromatin were either lyophilized or precipitated in $25 \%(\mathrm{v} / \mathrm{v})$ trichloroacetic acid (TCA), pelleted at $15000 \mathrm{~g}$, washed with $100 \%$ acetone and vacuum-dried. Samples were solubilized in sample buffer containing $4 \%$ SDS, $12 \%$ (w/v) glycerol, $50 \mathrm{~mm}$ Tris, $2 \%$ $(\mathrm{v} / \mathrm{v}) 2$-mercaptoethanol, $0.01 \%(\mathrm{w} / \mathrm{v})$ Servablue G, $\mathrm{pH} 6.8$, boiled and separated in a $17.6 \% \mathrm{SDS}-$ tricine-polyacrylamide resolution gel, with a $5 \%$ stacking gel (Schägger \& von Jagow, 1987).

\section{Staining and destaining procedures}

Coomassie brilliant blue. Gels were stained with $0.25 \%(\mathrm{w} / \mathrm{v})$ Coomassie brilliant blue R-250 in methanol:water:glacial acetic acid $(5: 5: 1)$ for $1 \mathrm{~h}$ and destained with methanol:water:glacial acetic acid $(4: 5: 1)$ for $1 \mathrm{~h}$ and $(10: 83: 7)$ overnight, respectively.

Differential destaining with $\mathrm{FeCl}_{3}$ of Coomassiestained histones. Differential destaining with $0 \cdot 1 \mathrm{M}$ $\mathrm{FeCl}_{3}$ in $20 \%(\mathrm{v} / \mathrm{v})$ ethanol was performed for $6 \mathrm{~h}$ (Spiker, Key \& Wakim, 1976; Duschak \& Cazzulo, 1990).

Bromophenol blue. Gels, fixed in methanol:water: acetic acid as described above were stained in $0.01 \%$ $(\mathrm{w} / \mathrm{v})$ bromophenol blue in water $(\mathrm{pH} \mathrm{3.0)}$ ) for $24 \mathrm{~h}$ at room temperature, and differentially destained in $40 \%$ (v/v) $n$-propanol for 24 h at $55^{\circ} \mathrm{C}$ (Duschak $\&$ Cazzulo, 1990).

\section{Micrococcal nuclease digestion and DNA agarose gel electrophoresis}

Isolated nuclei of $T . b$. brucei procyclic culture forms as well as of bloodstream forms were digested with micrococcal nuclease, at concentrations of $0 \cdot 1,0 \cdot 2$ and 0.4 units per $20 \mathrm{~A}_{260}$ of nuclear suspension at $37^{\circ} \mathrm{C}$ (Hewish \& Burgoyne, 1973). Aliquots were withdrawn after 1,4 and $10 \mathrm{~min}$, and the digestion stopped with $\mathrm{Na}_{2}$ EDTA (final concentration $2.5 \mathrm{~mm}$ ). DNA was prepared for gel electrophoresis, and samples of about $5 \mu \mathrm{g}$ DNA were separated on a horizontal $1.5 \%(\mathrm{w} / \mathrm{v})$ agarose gel or on a linear $5.5 \%$ polyacrylamide gel and stained with ethidium bromide according to methods described by Sambrook, Maniatis \& Fritsch (1989).

\section{RESULTS}

\section{Effect of gentamicin on procyclic culture forms}

Procyclic culture forms are routinely cultivated in the presence of $10 \mu \mathrm{g}$ gentamicin $/ \mathrm{ml}$ of culture medium. In bacteria the antibiotic substance gentamicin interferes with mRNA translation and therefore leads to the synthesis of defective proteins. No influence of the antibiotic on the composition, structure and compaction of the chromatin could be seen, either in the absence of gentamicin or with a 5fold higher concentration of gentamicin than is commonly used $(50 \mu \mathrm{g} / \mathrm{ml}$ ) (results not shown).

\section{Structure and compaction pattern of soluble chromatin}

Soluble chromatin of $T . b$. brucei bloodstream forms, centrifuged through a sucrose gradient containing $10 \mathrm{~mm} \mathrm{NaCl}$, dialysed against concentrations of 0 , 10 , 40 , or $100 \mathrm{mM} \mathrm{NaCl}$, and prepared for $\mathrm{EM}$, showed condensation at increasing ionic strength (Fig. 1E-H). The compaction was more pronounced as compared to procyclic culture forms (Fig. 1I-M). However, solenoids (30 $\mathrm{nm}$ fibres) which are typical for rat liver chromatin (Fig. 1A-D), were not formed. Free linker DNA could barely be seen in rat liver chromatin (Fig. 1A-C), but was clearly visible in chromatin from both trypanosome stages at low salt concentrations (Fig. 1E-F and $\mathrm{I}-\mathrm{L}$ ). The nucleosomes of the chromatin filaments of procyclic culture forms were irregularly spaced (Fig. 1I). This was also seen in bloodstream forms but to a lesser extent (Fig. 1E), while chromatin of rat liver never showed such an irregular arrangement (Fig. 1A).

\section{Triton acid urea $P A G E$}

In triton acid urea gels, which separate proteins according to their hydrophobicity, core histones of trypanosomes migrated in four main complexes a, b, c, $\mathrm{d}$ and in a fast migrating, hydrophilic e-complex (Fig. 2, Lanes 2 and 3). Apart from the proteins of the d-regions, which had similar hydrophobic properties to $\mathrm{H} 4$ of higher eukaryotes (Fig. 2, Lane 1), all the histones of trypanosomes differed from those of higher eukaryotes. No proteins of higher eukaryotes can be seen in the e-region. 

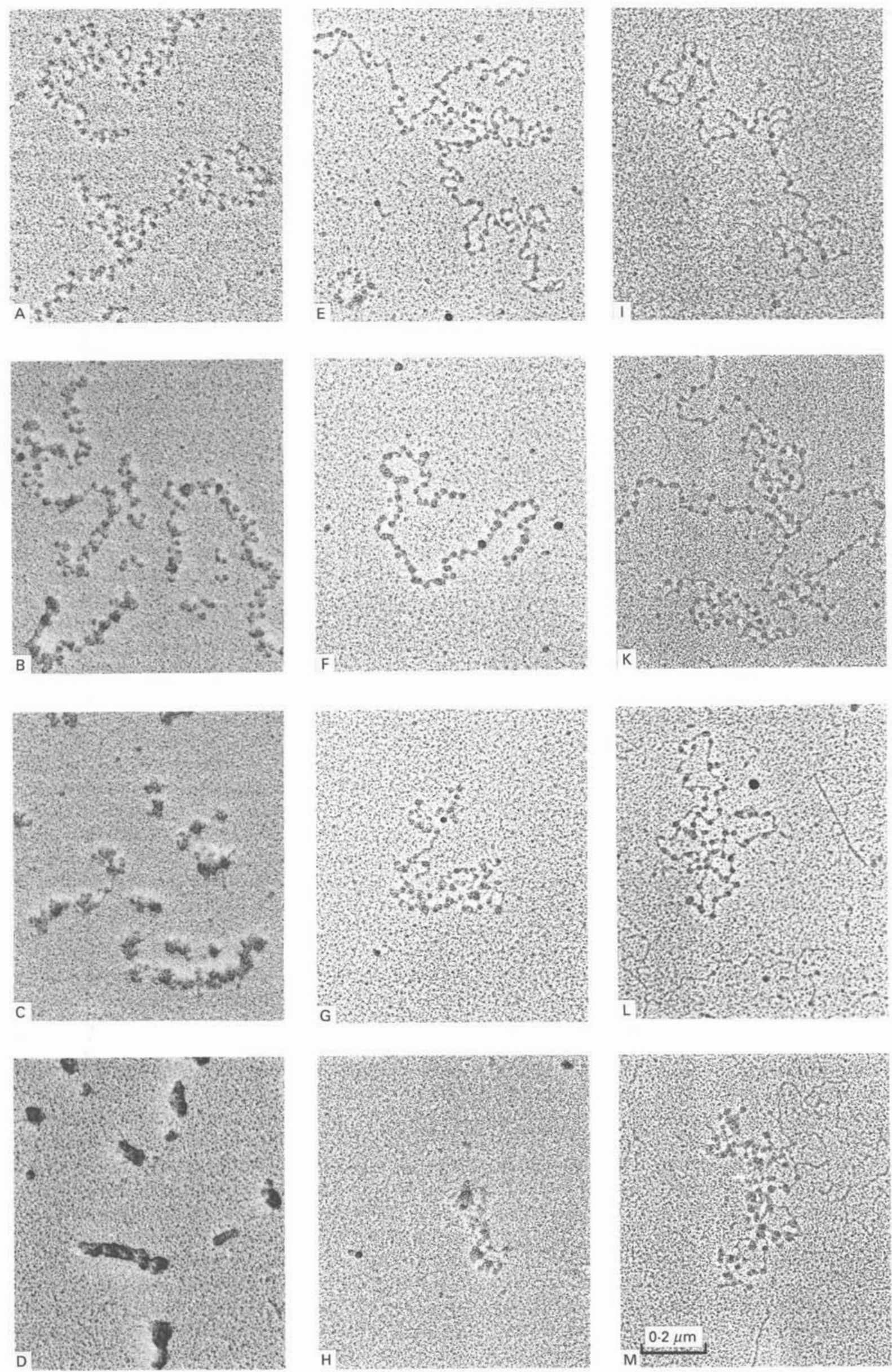

Fig. 1. For legend see opposite. 
Differences in the number of bands, their position in the gel and their relative amount were seen between the histones of the two trypanosome stages. This was especially true for the e-region (Fig. 2, Lanes 2 and 3 ). All the proteins in the e-area could be selectively extracted with $5 \%$ PCA (Fig. 2, Lanes 4 and 5). These proteins were metachromatically stained with Coomassie brilliant blue, which cannot be seen in black and white reproduction. Suspension of nuclei in $350 \mathrm{~mm} \mathrm{NaCl}$ extracted non-histone proteins but not histone $\mathrm{H} 1$ of rats (Fig. 2, Lane 8). Proteins of the e-region were not extracted from nuclei with $350 \mathrm{mM} \mathrm{NaCl}$, neither from procyclic culture forms (Fig. 2, Lane 6) nor from bloodstream forms (Fig. 2, Lane 7).

\section{Selective destaining of histones}

Histones of bloodstream forms, separated in Triton acid urea gels, were stained with $0.01 \%$ bromophenol blue, and the destaining of the proteins in $40 \% n$-propanol was densitometrically analysed (Fig. 3A). Selective destaining of the proteins in the e-region could be seen after $6 \mathrm{~h}$ (Fig. 3B). The proteins in the $b$ - and c-regions were destained after $24 \mathrm{~h}$, the staining intensity of the d-region was reduced, and no destaining took place in the a-region (Fig. 3C). Procyclic culture forms showed the same destaining behaviour (not shown). Differential destaining of Coomassie brilliant blue-stained histones with $0 \cdot 1 \mathrm{M} \mathrm{FeCl}_{3}$ revealed a selective destaining of the e-region after $2 \mathrm{~h}$ (not shown).

\section{SDS-tricine-PAGE}

Histones of higher eukaryotes separated into a complex of 4 core histones and $2 \mathrm{H} 1$ variants in SDS-tricine-PAGE (Fig. 4, Lane 1). Core-histones of trypanosomes and higher eukaryotes could be seen to differ in their electrophoretic mobility (Fig. 4, Lanes 1, 2 and 3). The strongest differences existed between rat liver histones $\mathrm{H} 3$ and $\mathrm{H} 4$ and their trypanosome counterparts a and $d$. Histones of bloodstream forms showed a different banding pattern compared to that of procyclic culture forms. In this high-resolution gel system, the histone $b$ was separated into two variants in both trypanosome stages (b1 and b2) (Fig. 4, Lanes 2 and 3) and histone c shows three variants $(\mathrm{c} 1, \mathrm{c} 2$ and $\mathrm{c} 3)$, but only in bloodstream forms (Fig. 4, Lane 3). Gels stained with Coomassie brilliant blue showed differences in the amounts of proteins b1 and b2 in procyclic culture forms and bloodstream forms (Fig. 4, Lanes
2 and 3). Neither procyclic culture forms nor bloodstream forms had any protein migrating in the H1 region of higher eukaryotes (Fig. 4, Lanes 1, 2 and 3 ). Proteins extracted with $5 \%$ PCA migrated in the lower region of the core-histones and were metachromatically stained with Coomassie brilliant blue (Fig. 4, Lanes 4 and 5). These PCA-extractable proteins separated in four bands $(1-4)$ in procyclic culture forms and in three bands $\left(1^{\prime}-3^{\prime}\right)$ in bloodstream forms.

Among the $\mathrm{HCl}$-extractable proteins of procyclic culture forms, only band 1 was clearly separated from the others, while bands 2-4 were partly overlaid by histone d. Only faint bands above and below that of histone $d$ indicate their presence (Fig. 4, Lane 6). Positioning of the bands by densitometric tracing revealed that the PCA-extractable bands $1^{\prime}, 2^{\prime}$ and $3^{\prime}$ of bloodstream forms were also visible in $\mathrm{HCl}$ extracts.

No significant differences could be seen between the two stages in proteins extractable in $0.25 \mathrm{M} \mathrm{HCl}$ or DNA-associated proteins derived from soluble chromatin (Fig. 4, Lanes 2, 3, 6 and 7).

\section{Nucleosomal pattern after micrococcal nuclease digestion}

Ladders of DNA fragments ranging from oligonucleosomes to mononucleosomes were visible in agarose gels when chromatin of $T . b$. brucei procyclic culture forms were digested with micrococcal nuclease for $1 \mathrm{~min}$ (Fig. 5A, Lane 3). Chromatin of bloodstream forms was digested to a lesser extent, and that of rat liver still less (Fig. 5A, Lanes 4 and 1). After $10 \mathrm{~min}$ digestion, nucleosome ladders were still found for rat liver (Fig. 5A, Lane 9), whereas chromatin of both trypanosome stages was more extensively digested, mainly to mononucleosomes and dinucleosomes (Fig. 5A, Lanes 7 and 8). The strongest differences in the digestion patterns between procyclic culture forms and bloodstream forms were seen after 4 min (Fig. 5A, Lanes 5 and 6). Mixed preparations of DNA fragments of procyclic culture forms and bloodstream forms after $1 \mathrm{~min}$ digestion (Fig. 5A, Lane 2) showed nucleosomal ladders identical to those of single preparations (Fig. $5 \mathrm{~A}$, Lanes 3 and 4), which demonstrates that the linker length is identical for both life-cycle stages of $T . b$. brucei. The differences were not affected by changes in the enzyme concentration $(0 \cdot 1,0 \cdot 2$ and $0 \cdot 4$ units micrococcal nuclease per $20 \mathrm{~A}_{260}$ nuclei).

The same samples separated on a high-resolution polyacrylamide gel showed mainly mononucleo-

Fig. 1. Soluble chromatin of Trypanosoma brucei brucei and of rat liver from a sucrose gradient containing $10 \mathrm{~mm}$ $\mathrm{NaCl}$, dialysed against concentrations of $0 \mathrm{~mm} \mathrm{NaCl}(\mathrm{A}, \mathrm{E}, \mathrm{I}), 10 \mathrm{~mm} \mathrm{NaCl}(\mathrm{B}, \mathrm{F}, \mathrm{K}), 40 \mathrm{~mm} \mathrm{NaCl}(\mathrm{C}, \mathrm{G}, \mathrm{L})$ and $100 \mathrm{~mm} \mathrm{NaCl}(\mathrm{D}, \mathrm{H}, \mathrm{M})$. Rat liver (A-D); bloodstream forms $(\mathrm{E}-\mathrm{H})$; procyclic culture forms $(\mathrm{I}-\mathrm{M})$. 

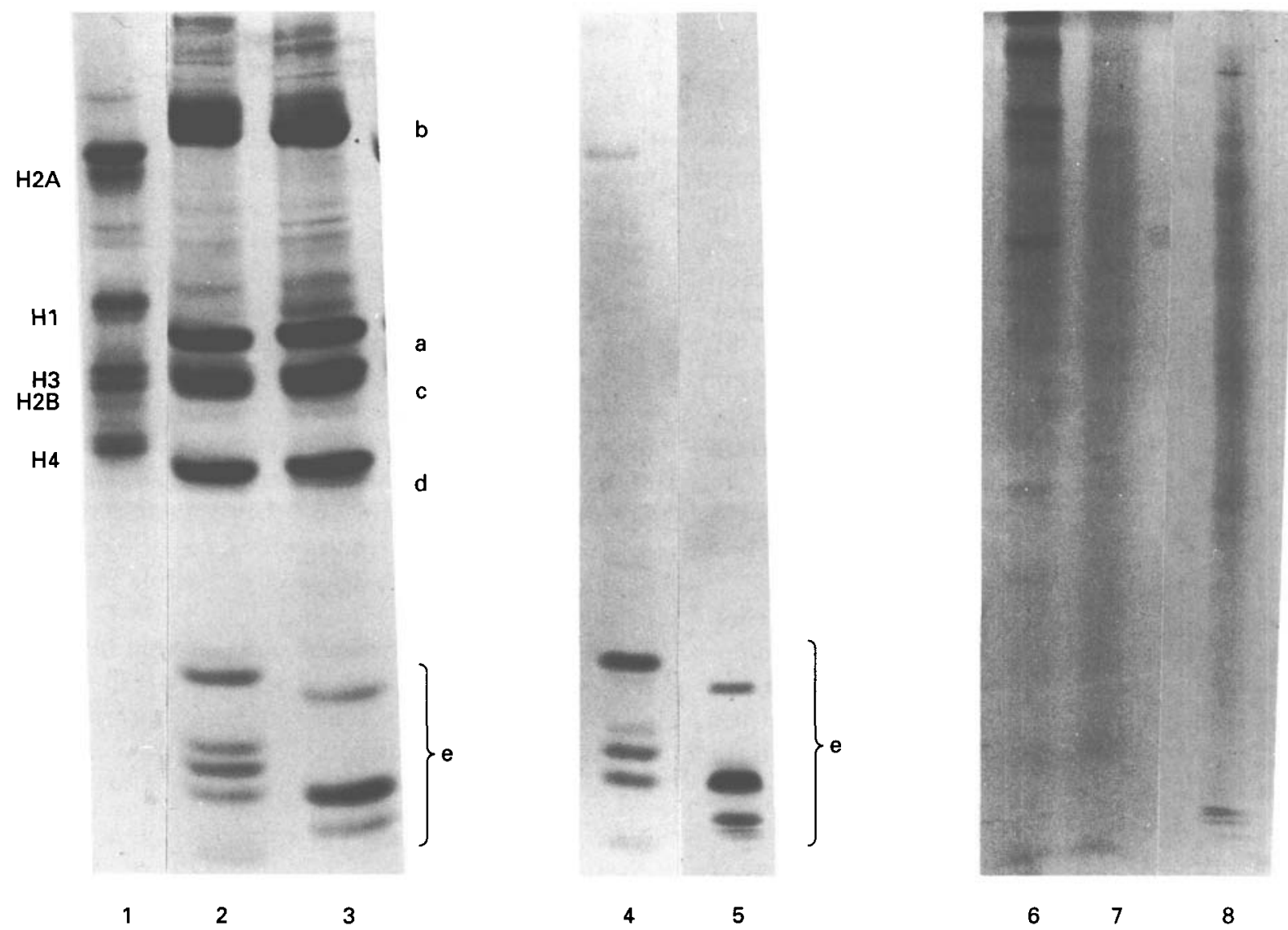

Fig. 2. Histone analysis in Triton acid urea PAGE. Lane 1: calf thymus histones. HCl-extracted histones of nuclei of Trypanosoma brucei brucei procyclic culture forms (Lane 2) and of bloodstream forms (Lane 3); $5 \%$ perchloric acidextracted histones of procyclic culture forms (Lane 4) and bloodstream forms (Lane 5). Non-histone proteins extracted with $350 \mathrm{~mm} \mathrm{NaCl}$ from procyclic culture forms (Lane 6), bloodstream forms (Lane 7) and rat liver (Lane 8).

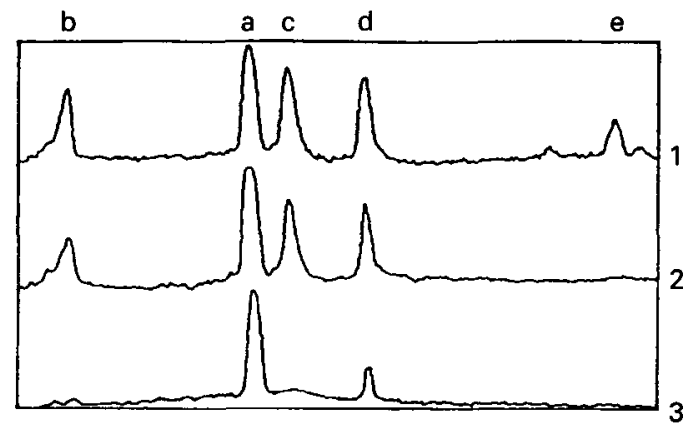

Fig. 3. Selective destaining of bromophenol blue stained histones. Histones of Trypanosoma brucei brucei bloodstream forms, separated on a Triton DF-16 acid urea gel and stained with bromophenol blue, were treated with $40 \% n$-propanol, which destains selectively histone $\mathrm{H} 1, \mathrm{H} 2 \mathrm{~A}$ and $\mathrm{H} 2 \mathrm{~B}$. Densitometric Trace 1 :

before destaining; Trace 2: after $6 \mathrm{~h}$; Trace 3 after $24 \mathrm{~h}$ of destaining. After $6 \mathrm{~h}$ the e-bands and after $24 \mathrm{~h}$ the $\mathrm{b}$ and c-bands, which are the counterparts of $\mathrm{H} 2 \mathrm{~A}$ and $\mathrm{H} 2 \mathrm{~B}$ in higher eukaryotes, are destained.

some-sized and traces of dinucleosome-sized DNA fragments in procyclic culture forms after digestion for $10 \mathrm{~min}$ (Fig. 5B, Lane 2). Bloodstream forms showed fragments up to four nucleosomes at the same digestion time (Fig. 5B, Lane 3). The mean size of the mononucleosome fragments was larger for the bloodstream forms than for procyclic culture forms after $10 \mathrm{~min}$ digestion. The centre of the corresponding band in bloodstream forms was found above the $154 \mathrm{bp}$ fragment of the DNA marker, while the centre of the band of the procyclic culture forms was situated in the region of this marker fragment. After 30 min digestion, DNA fragments were found in the region of $154 \mathrm{bp}$ as well as below this marker for both trypanosome stages (Fig. 5B, Lanes 4 and 5).

\section{DISCUSSION}

The identification of histone $\mathrm{H} 1$ can be difficult, since it is the most variable of all histones. In several cases, the original reports stated that $\mathrm{H} 1$ was absent (van Holde, 1989) and only careful choice of experimental conditions, use of protease inhibitors and specially adapted gel systems allowed the demonstration of its presence. In T.b.brucei, a histone $\mathrm{H} 1$-like protein was postulated to be absent (Hecker \& Gander, 1985; Hecker et al. 1989; Bender 

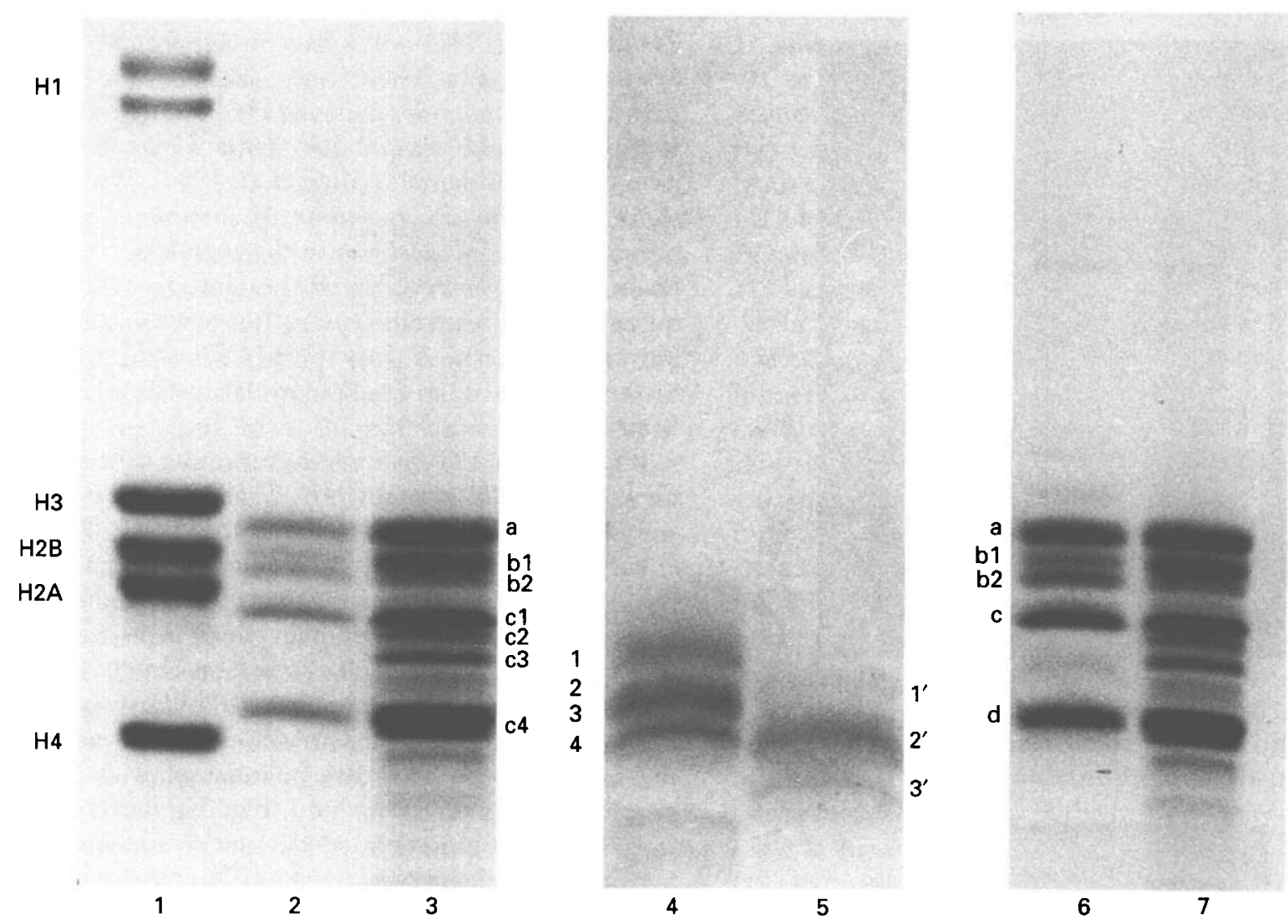

Fig. 4. Histone analysis in SDS-tricine-PAGE. Lane 1: calf thymus histones. HCl-extracted histones of Trypanosoma brucei brucei procyclic culture forms (Lane 2) and bloodstream forms (Lane 3); $5 \%$ perchloric acid-extracted histones of bloodstream forms (Lane 4) and of procyclic culture forms (Lane 5); histones from soluble chromatin of procyclic culture forms (Lane 6) and bloodstream forms (Lane 7).

et al. $1992 a-d)$, although it was shown in other trypanosomatids (Toro \& Galanti, 1988, 1990; Duschak \& Cazzulo, 1990).

In the present paper, proteins called e-region were seen in $T . b$. bruce $i$ for the first time. We postulate that the proteins of the e-region are histone $\mathrm{H} 1$-like proteins. This is supported by their extractability with $5 \%$ PCA, a typical feature of histone $\mathrm{H} 1$ of higher eukaryotes (Sanders, 1977). If these proteins are stained with Coomassie Brilliant blue, they show metachromasia; a reddish colour, which is a sign of a high lysine content (Duhamel, Meezan \& Brendel, 1980). Additional evidence comes from destaining experiments with $n$-propanol (Duschak \& Cazzulo, 1990) and $\mathrm{FeCl}_{3}$ (Spiker et al. 1976; Duschak \& Cazzulo, 1990), which destains histone H1. Using both methods the e-region is destained first. Additionally, due to their non-extractability with $350 \mathrm{~mm} \mathrm{NaCl}$, it could be ruled out that these proteins were non-histones (Johns, 1982). Antibodies directed against $\mathrm{H} 1$ of sea urchin showed cross-reactivity to the fast migrating proteins of $T$. cruzi (Toro \& Galanti, 1988), the counterparts of the e-bands in T. b. brucei. It has been shown by means of amino acid analysis that these proteins of $T$. cruzi are histone H1-like proteins (G. C. Toro \& N. Galanti, personal communication).
Triton binds to histones proportionally to their degree of hydrophobicity and thereby reduces the electrophoretic mobility (Hardison \& Chalkley, 1978). The histone H1-like proteins of T.b.brucei are strongly hydrophilic and very fast migrating in Triton acid urea gels. The banding pattern in Triton DF-16 gels of histones of T.cruzi, T.b.brucei and Crithidia fasciculata is similar and all species possess fast migrating hydrophilic histone $\mathrm{H} 1$-like proteins. However, the banding pattern of the fast migrating bands in trypanosomatids differs. Differences in the number, amount and position of the $\mathrm{H} 1$-like proteins can also be seen, when the two stages of the life-cycle of $T . b$. brucei are compared.

Histone H1-like proteins of T. b. brucei run out of Triton and acid urea gels owing to their high mobility unless specially adapted systems are used. In SDS gels they can only be detected if the linear SDS-tricine-PAGE according to the method of Schägger \& von Jagow (1987), optimized for highest resolution in the region of the core histones, is used. The resolution in this gel system is superior to that in gradient gels according to Laemmli (1970) which were originally used by Hecker \& Gander (1985), and Bender et al. (1992a). The strong hydrophilic character and the small molecular weight of the H1like proteins of T.b. brucei are the reasons why 
A

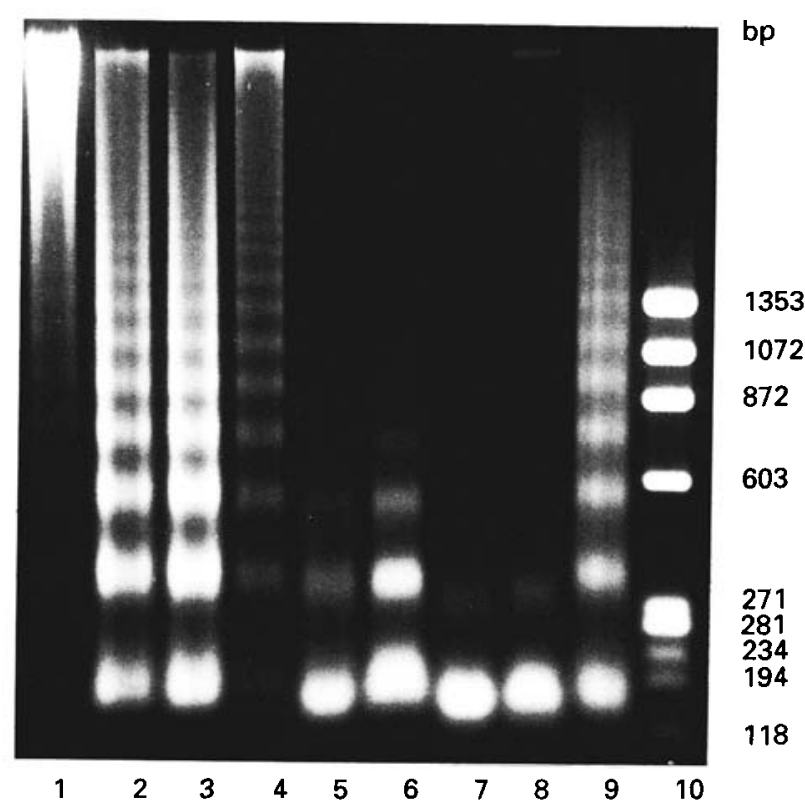

B

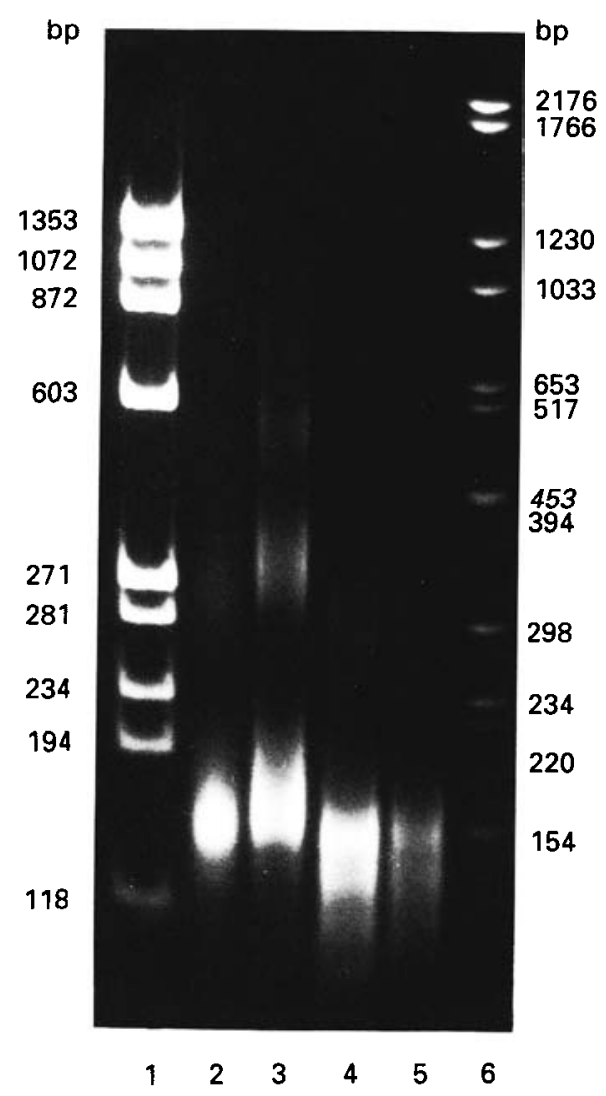

Fig. 5. (A) Separation of DNA fragments in a $1.5 \%$ agarose gel. Micrococcal nuclease digestion patterns of chromatin of rat liver, Trypanosoma brucei brucei procylic culture and bloodstream forms. Marker: HAE III fragments of $\Phi$ X 174 RF DNA (Lane 10). Lanes 1 and 9: rat liver; Lanes 3,5 and $7:$ procyclic culture forms; Lanes 4, 6 and 8: bloodstream forms; Lane 2: mixture of procyclic culture and bloodstream forms. Digestion times: Lanes 1-4: $1 \mathrm{~min}$; Lanes 5 and 6: 4 min; Lanes 7-9: $10 \mathrm{~min}$. (B) Separation of DNA
Hecker \& Gander (1985), Hecker et al. (1989) and Bender et al. (1992a-d), failed to demonstrate their existence.

In higher eukaryotes, histone $\mathrm{H} 1$ is composed of a central hydrophobic globular domain and two adjacent hydrophilic tails (Allan et al. 1980). We could show that the PCA extracted histone H1-like proteins of $T . b$. bruce $i$ run in the region of the core histones in SDS-PAGE and therefore they may be quite small. This finding is in line with a study of Hayashi, Hayashi \& Iwai (1987) who described a histone $\mathrm{H} 1$ with a small globular part in Tetrahymena.

If the $\mathrm{N}$ - and C-terminal hydrophilic tails of the histone $\mathrm{H} 1$-like proteins in T.b.brucei are of a similar length, compared to those of higher eukaryotes, then the hydrophobic globular region would occupy only a small proportion. The globular part of histone $\mathrm{H} 1$ is the domain which is responsible for the binding to the nucleosome (van Holde, 1989). A small sized globular domain could indicate a weak interaction of the trypanosome $\mathrm{H} 1$-like proteins with the nucleosomes. The core histones of $T . b$. bruce procyclic culture forms and bloodstream forms, separated according to the molecular weight or to the hydrophobicity, are similar, but differ in the position and number of the bands as compared to higher eukaryotes. This result is in line with differences reported for the amino acid composition between T.b. brucei procyclic culture forms and higher eukaryotes (Bender et al. 1992a). By selective destaining with $n$-propanol, we were able to show that the proteins of the bloodstream forms, which migrate in the positions of histones $b$ and $c$ in Triton gels are the counterparts of $\mathrm{H} 2 \mathrm{~A}$ and $\mathrm{H} 2 \mathrm{~B}$ (Duschak \& Cazzulo, 1990). Life-cycle specific variants and/or modifications can be seen in histone $b$, which is resolved in two proteins b1 and b2 in SDS-tricinePAGE, as well as in histone c, which is resolved only in bloodstream forms into three bands $\mathrm{c} 1, \mathrm{c} 2$ and $\mathrm{c} 3$. Differences in the amount between the two bands of protein b occur between the two trypanosome stages and can be seen in SDS-tricine as well as in Triton acid urea gels.

Weak interaction and instability within the chromatin enhances the accessibility of the linker DNA towards micrococcal nuclease and this leads to a

fragments in a $5.5 \%$ polyacrylamide gel. Markers: HAE III fragments of $\Phi$ X 174 RF DNA (Lane 1) and DNA fragments of pBR328 cleaved with $B g l \mathrm{I}$ and $H$ inf I (Lane 6). Procyclic culture forms (Lanes 2 and 4) and bloodstream forms (Lanes 3 and 5) after 10 or $30 \mathrm{~min}$ digestion. The centre of the band of mononucleosome fragments of bloodstream forms (Lane 3 ) is above the $154 \mathrm{bp}$ marker fragments (Lane 6), while one of the procyclic culture forms (Lane 2) is in the region of the latter. 
faster digestion of the chromatin of the procyclic culture forms (Hecker et al. 1989) relative to higher eukaryotes. Bloodstream forms appear to be intermediate. Digestion is slower than in procyclic culture forms, but still faster than for higher eukaryotes. There is a clear digestion barrier in higher eukaryotes in the presence of H1 (Telford \& Stewart, 1989), which cannot be seen in procyclic culture forms (Hecker et al. 1989). After digestion for $10 \mathrm{~min}$, bloodstream forms yield larger mononucleosome-sized DNA fragments than the procyclic culture forms. These larger fragments are no longer seen after $30 \mathrm{~min}$ digestion. A weak digestion barrier in bloodstream forms may be due to a somewhat stronger interaction between histone $\mathrm{H} 1$-like proteins and the DNA, as compared to the procyclic culture form.

Genetically inactive chromatin of higher eukaryotes forms $30 \mathrm{~nm}$ fibres (solenoids) at $100 \mathrm{~mm}$ $\mathrm{NaCl}$. The formation of the $30 \mathrm{~nm}$ fibres depends on the presence of the lysine-rich histone $\mathrm{H} 1$. In the absence of histone $\mathrm{H} 1$, nucleosome filaments do not condense to solenoids, and form only loose aggregates (Thoma et al. 1979). The spacing of the nucleosomes on the DNA is irregular and nucleosome sliding occurs (Stein \& Bina, 1984).

Chromatin of $T . b$. brucei procyclic culture forms, prepared under identical conditions to rat liver chromatin, is organized in the form of nucleosome filaments. Previous studies on the ultrastructure of the chromatin of $T . b$. brucei procyclic culture forms revealed a bad conservation of the nucleosome filaments (Hecker \& Gander, 1985). The nucleosomes were irregularly spaced and at increasing ionic strength only a slight aggregation of the nucleosomes could be seen (Hecker \& Gander, 1985). The interactions of histones and DNA are weak and are easily destabilized by experimental manipulation (Hecker \& Gander, 1985; Hecker et al. 1989; Bender et al. $1992 c)$.

To try to improve the preservation of the chromatin filaments of trypanosomes, the effect of increasing the concentration of glutaraldehyde for the fixation of soluble chromatin for EM studies was investigated. The results obtained with $0.2 \%$ glutaraldehyde were superior to those with the 'conventional' concentration of $0.1 \%$ (Thoma et al. 1979; Hecker \& Gander, 1985). Chromatin prepared with $0.5 \%$ glutaraldehyde showed an identical condensation behaviour to the one fixed with $0.2 \%$.

The stability of chromatin depends on the nature and strength of the DNA-protein interactions (Yager, McMurray \& van Holde, 1989). In contrast to the chromatin of procyclic culture forms, the chromatin of bloodstream forms shows a stronger condensation at increasing ionic strength but it does not form $30 \mathrm{~nm}$ fibres. The compaction pattern of soluble chromatin of $T . b$. brucei bloodstream forms is structurally similar to that of T. cruzi epimastigote culture forms (Hecker \& Gander, 1985). Histone H1-like proteins were shown for T. cruzi by Toro $\&$ Galanti (1988, 1990). Hecker \& Gander (1985) could induce a higher condensation level in $T$. cruz $i$ with the addition of a histone $\mathrm{H} 1$-containing fraction from rat liver chromatin. This result suggested an $\mathrm{H} 1$ binding site in $T$. cruzi chromatin. Additionally, Bender (1991) showed that a binding site for histone H1 exists in the chromatin of T.b. brucei procyclic culture forms.

The finding of Hecker $\&$ Gander (1985), that no higher condensation level was achieved in procyclic culture forms of $T . b$. bruce $i$ by the addition of $\mathrm{H} 1$ of rats, is most probably due to the damage of the chromatin having been introduced by the experimental procedure to make soluble chromatin and the concomitant dissociation of nucleosomes from the DNA. A subsequent reconstitution is no longer possible. The result is the formation of 'clumps on a string' with large stretches of free DNA.

The histone patterns of the e-region of procyclic culture forms and bloodstream forms are different in number and position of bands and in their relative amounts. Modifications of the histones may influence histone-histone interactions as shown by Simpson (1981) for higher eukaryotes, and may contribute to a higher stability of the core particle especially in bloodstream forms. Effects of histone modifications were shown on the condensation behaviour of nucleosome filaments into higher-order structures for higher eukaryotes (Marion et al. 1985).

The different histone patterns of the two compared trypanosome stages are most likely the reason for the different condensation behaviour of their chromatin. The H1-like proteins of procyclic culture forms seem to interact very weakly with the DNA and this could be the reason why no condensation takes place, since most of these proteins dissociate during the preparation of soluble chromatin. This would also explain the poor preservation of the nucleosome filaments of procyclic culture forms at $0 \mathrm{~mm} \mathrm{NaCl}$, which is in accordance with the results of Hecker et al. (1989), who showed that chromatin of procyclic culture forms is very unstable. As chromatin from both stages of the life-cycle was prepared under identical conditions, observed differences of the chromatin parameters must be intrinsic differences between the life-stages of T.b. brucei.

We wondered why trypanosomes undergo changes in their histones during their life-cycle and suggest that the stronger DNA-histone interaction in bloodstream forms might be a requirement for a stabilization of the chromatin at the higher temperatures of the mammalian host. When procyclic culture forms were cultivated above $35^{\circ} \mathrm{C}$, they stopped cell division and died after approximately 3 days. All chromatin parameters investigated, including the histone pattern in gels, were those usually found in procyclic culture forms. The fact that procyclic 
culture forms cannot produce the histone $\mathrm{H} 1$ pattern and core histone variants of modifications of bloodstream forms and die after several days when cultivated between 35 and $37^{\circ} \mathrm{C}$, supports this hypothesis. Since trypanosomes derive from freeliving flagellates (Lumsden \& Evans, 1976), the ability of bloodstream forms to support higher temperatures might be regarded as an adaptation to parasitism.

It can be concluded that $T . b$. brucei contains a full set of histones, including $\mathrm{H} 1$-like proteins, and that the poor condensation of its chromatin is not due to the absence of $\mathrm{H} 1$, but most probably to peculiar properties of $\mathrm{H} 1$ and core histones. Despite common features, the histones and therefore various properties of the nuclear chromatin are quite different between bloodstream and procyclic culture forms. As a consequence, the latter can no longer be regarded as the only model for all stages of the lifecycle of $T . b$. brucei.

We would like to thank Jennifer Jenkins and Patrick Lorenz for the careful reading of the manuscript, Ruth Nuesch for the preparation of the culture medium and Irène Herde for skilful technical assistance.

\section{REFERENCES}

ALFAGEME, C. R., ZWEIDLER, A., MAHOWALD, A. \& COHEN, L. H. (1974). Histones of Drosophila embryos. Fournal of Biological Chemistry 249, 3729-36.

Allan, J., hartman, P. G., CRANE-Robinson, C. \& AVILES, F. X. (1980). The structure of histone $\mathrm{H} 1$ and its location in chromatin. Nature, London 288, 675-9.

BENDER, K. (1991). Biochemical and structural aspects of the nuclear chromatin of procyclic Trypanosoma brucei brucei. Ph.D. thesis, University of Basel.

BENDER, K., BETSCHART, B., SCHALLER, J., KÄMPFER, U. \& HECKER, H. (1992a). Biochemical properties of histonelike proteins of procyclic Trypanosoma brucei brucei. Acta Tropica 50, 169-84.

BENDER, K., BETSCHART, B., SCHALLER, J., KÄMPFER, U. \& HECKER, H. (1992b). Sequence differences between histones of procyclic Trypanosoma brucei brucei and higher eukaryotes. Parasitology 105, 97-104.

BENDER, K., BETSCHART, B. \& HECKER, H. (1992c). Histone-DNA interactions in the chromatin of procyclic Trypanosoma brucei brucei. Parasitology Research 87, 495-500.

BENDER, K., BETSCHART, B., MaRion, C., Michalon, P. \& HECKER, H. (1992d). Structural differences between the chromatin of procyclic Trypanosoma brucei brucei and higher eukaryotes as probed by immobilized trypsin. Acta Tropica 52, 69-78.

BRUN, R. \& SCHÖNENBERGER, M. (1979). Cultivation and in vitro cloning of Trypanosoma brucei in a semi-defined medium. Acta Tropica 36, 289-92.

DUHAMEL, R. C., MEEZAN, E. \& BRENDEL, K. (1980).

Metachromatic staining with Coomassie-Brilliant-Blue R-250 of the proline-rich calf thymus histone $\mathrm{H} 1$. Biochimica et Biophysica Acta 626, 432-42.
DUSCHAK, V. G. \& CAzzulo, J.J. (1990). The histones of the insect trypanosomatid, Crithidia fasciculata. Biochimica et Biophysica Acta 1040, 159-66.

ELPIDINA, E. N., ZAITSEVA, G. N. \& KRASHENINNIKOV, J. A. (1979). Histones from Trypanosoma lewisi nuclei. Biokhimiya 44, 1830-41.

HAMM, B., SCHINDLER, A., MECKE, D. \& DUSZENKO, D. (1990). Differentiation of Trypanosoma brucei bloodstream trypomastigotes from long slender to short stumpy-like forms in axenic culture. Molecular and Biochemical Parasitology 40, 13-22.

HARdison, R. \& CHALKLEY, R. (1978). Polyacrylamide gel electrophoretic fractionation of histones. Methods in Cell Biology 17, 235-51.

hayashi, T., HaYAShI, H. \& IWAI, K. (1987). Tetrahymena histone $\mathrm{H} 1$. Isolation and amino acid sequence lacking the central hydrophobic domain conserved in other H1 histones. Fournal of Biochemistry 102, 369-76.

HECKER, H. \& GANDER, E. s. (1985). The compaction pattern of the chromatin of trypanosomes. Biology of the Cell 53, 199-208.

HECKER, H., BENDER, K., BETSCHART, B. \& MODESPACHER, U. P. (1989). Instability of the nuclear chromatin of procyclic Trypanosoma brucei brucei. Molecular and Biochemical Parasitology 37, 225-34.

HEWISH, D. R. \& BURGOYNE, L. A. (1973). Chromatin substructure. The digest of chromatin DNA at regularly spaced sites by a nuclear deoxyribonuclease. Biochemical and Biophysical Research Communication 52, 504-10.

JohmanN, C. A. \& GOROvsky, M. A. (1976). Purification and characterisation of histones associated with the macronucleus of Tetrahymena. Biochemistry $\mathbf{1 5}$, $1249-56$

JoHns, E. W. (1982). Chapter 1. In The $H M G$ Chromosomal Proteins (ed. Johns, E. W.), pp. 1-7. New York: Academic Press.

LAEMMLI, U. K. (1970). Cleavage of structural proteins during the assembly of the head of bacteriophage $\mathrm{T} 4$. Nature, London 227, 680-5.

LANHAM, S. M. \& GODFREY, D. G. (1970). Isolation of salivarian trypanosomes from man and other mammals using DEAE-Cellulose. Experimental Parasitology 28, 521-34.

LUMSDEN, W. H. R. \& EVANS, D. A. (1976). Biology of the Kinetoplastida, Vol. I. New York: Academic Press.

MARION, C., MARTINAGE, A., TIRARD, A., ROUX, B., DaUNe, M. \& MAZEN, A. (1985). Histone phosphorylation in native chromatin induces local structural changes as probed by electric birefringence. Fournal of Molecular Biology 186, 367-79.

NOLL, H. (1969). An automatic high-resolution gradient analyzing system. Analytical Biochemistry 27, 130-49.

SAMBROOK, J., MANIATIS, P. T. \& FRITSCH, E. F. (1989). Molecular Cloning. A Laboratory Manual. Cold Spring Harbor, New York: Cold Spring Harbor Laboratory. SANDERS, C. (1977). A method for the fractionation of the high-mobility group non-histone proteins. Biochemical and Biophysical Research Communications 78, 1034-42. SCHÄGGER, H. \& VON JAGOW, G. (1987).

Tricine sodium-dodecyl sulfate polyacrylamide gel electrophoresis for the separation of proteins in the range from 1 to $100 \mathrm{kDa}$. Analytical Biochemistry 166, 368-79. 
ShaPIRO, S. Z. \& DOXSEY, S. J. (1982). Purification of nuclei from a flagellate protozoan, Trypanosoma brucei. Analytical Biochemistry 127, 112-15.

Simpson, R. T. (1981). Modulation of nucleosome structure by histone subtypes in sea urchin embryos. Proceedings of the National Academy of Sciences, USA 78, 6803-7.

SPIKER, S., KEY, J. L. \& WAKIM, B. (1976). Identification and fractionation of plant histones. Archives of Biochemistry and Biophysics 176, 510-18.

STEIN, A. \& BINA, M. (1984). A model chromatin assembly system: factors affecting nucleosome spacing. Fournal of Molecular Biology 178, 341-63.

TELFORD, D. J. \& STEWART, B. W. (1989). Micrococcal nuclease: its specificity and use for chromatin analysis. International fournal of Biochemistry 21, $127-37$.

THOMA, F., KOLLER, TH. \& KLUG, A. (1979). Involvement of histone $\mathrm{H} 1$ in the organization of the nucleosome and the salt-dependent superstructures of chromatin. Fournal of Cell Biology 83, 403-24.

THOMA, F. \& KOLLER, TH. (1981). Unravelled nucleosomes, nucleosome beads and higher-order structures of chromatin: influence of non-histone components and histone H1. Fournal of Molecular Biology 149, 709-33.

TORO, G. C. \& GALANTI, N. (1988). H1 histone and histone variants in Trypanosoma cruzi. Experimental Cell Research 174, 16-24.

TORO, G. C. \& GALANTI, N. (1990). Trypanosoma cruzi histones. Further characterization and comparison with higher eukaryotes. Biochemistry International 21, 481-90.

TORO, G. C., WERNSTEDT, C., MEDiNA, C., JARAMILlo, N., HELLMANN, U. \& GaLANTi, N. (1992). Extremely divergent histone- $\mathrm{H} 4$ sequence from Trypanosoma cruzi-evolutionary implications. Fournal of Cellular Biochemistry 49, 266-71.

VAN HOLdE, K. E. (1989). Chromatin. In Springer Series in Molecular Biology (ed. Rich, A.), pp. 168-80 and pp. 317-43. New York: Springer Verlag.

VICKERMAN, K. \& PRESTON, T. M. (1970). Spindle microtubules in the dividing nuclei of trypanosomes. Fournal of Cell Science 6, 365-83.

YAGER, T. D., MCMURRAY, T. \& VAN HOLDE, K. E. (1989). Salt-induced release of DNA from nucleosome core particles. Biochemistry 28, 2271-81. 